\title{
Towards Safety in Human Robot Interaction
}

\author{
Guido Herrmann · Chris Melhuish
}

Accepted: 12 May 2010 / Published online: 21 May 2010

(C) Springer Science \& Business Media BV 2010

Many new application areas are emerging where robots will be required to co-operate with or assist humans. This means that they are sharing the same work-space or they are working in our 'personal' space. The fundamental resultant issue of working in co-located space is that of safety. In service and social robotics, a major focus has therefore to be safe and reliable operation. It has been identified by some researchers that safety might be expressed in two inter-related modes, namely: physical and behavioural. Physical Safety is quite well understood and refers to potential failures in such areas as torque control and sensor failure as well as exploiting such phenomena as passive compliance. Behavioural Safety is a relatively new research theme, which aims to exploit the bi-directional multi-modal communication channels employed by humans such as: gesture, body pose, facial expressions, back-channel utterances and speech. This further requires individual and shared cognitive models between robot and human to provide the basis for models of shared goals and shared intentions. The key hypothesis is that safe interaction between human and robot can be engineered physically and cognitively for joint physical tasks requiring co-operative manipulation of real world objects. The advancement of actuator technology, mechanical systems design, sensor systems, computational power, control technology and strong links with disciplines such as cognitive psychology has lead us to this assumption to be true in

G. Herrmann ( $\square)$

Bristol Robotics Laboratory and the Department of Mechanical Engineering, University of Bristol, Bristol, UK

e-mail: g.herrmann@bristol.ac.uk

C. Melhuish

Bristol Robotics Laboratory, University of West England Bristol and University of Bristol, Bristol, UK

e-mail: Chris.Melhuish@brl.ac.uk future. Considering this hypothesis as guiding principle, this special issue addresses a significant issue of current modern social robotics.

Many of the papers published here in this special issue on safety are the result of close international collaboration and communication, in particular in Europe. Significant European projects such as the CHRIS project (http://www.chrisfp7.eu/) have lead to close links between many of the institutions presenting their papers in this special issue. Nine papers are published from eleven different research institutions and most notably also from industrial parties, showing that safety in social robotics is of great concern to the whole science and engineering community. Thus, a wide range of topics on safe human-robot interaction (HRI) in social and service robotics is covered. The submissions can be classed into

1. Analysis and Design of Safety Aspects, providing a holistic view on the needs for direct human-robot interaction. This also includes direct insight from industrialists participating also in the standardization of safety requirements of human-robot interaction.

2. Design of robots ensuring safety via the correct development of the mechanical or actuator system.

3. Design of low- and medium level controllers to achieve safe compliance via direct force compliance.

4. High-level recognition, control and decision algorithms for human-robot interaction.

\section{Overview of the Papers}

In the first paper, the analysis of "Safety of interactive robotics-Learning from accidents" is carried out by Timo Malm, Juhani Viitaniemi, Jyrki Latokartano, Salla Lind, Outi Venho-Ahonen and Jari Schabel. Industrial experience 
of the authors on the use of robots in automation and production in Finland has lead to guidelines on the control and decisions to be undertaken to avoid human-robot interaction related accidents. For this, the authors have sourced from Finnish databases 25 severe accidents attributed to humanrobot interaction, showing that actions to diminish the effects of collision are important. It is also pointed out that current practice demands that upon detection of a collision, active movements are typically not allowed without human supervision. Moving a robot away to a safe position would entail some adjustments of the current safety requirements and standards.

The second paper titled "Towards the development of international safety standards for human robot interaction" and authored by Christopher Harper and Gurvinder Virk provides the necessary view on new safety standards for future human-robot interaction. It delivers a survey of the work being performed by the ISO committee TC184/SC2 "Robots and Robotic Devices". This committee is developing safety standards for robotic applications in personal and medical care, as well as revising existing industrial robot standards with requirements for new applications. A key driver of the new standards is the need for safety guidelines for human robot interaction, as the new applications involve much more extensive HRI behavior than previous generations of industrial robots.

In the third paper on "Safety Analysis for a HumanFriendly Manipulator" by Sami Haddadin, Alin AlbuSchäffer and Gerd Hirzinger, the German Aerospace Center (DLR) Lightweight Robot III (LWR-III) is investigated for possible sources of risks of injury in human-robot interaction. This risk is quantified via impact tests using standard automobile crash-test facilities. An analysis for softtissue injury based on swine experiments is carried out. An overview about the variety of investigations necessary to provide a safety analysis of a human-friendly robot based on biomechanical injury results.

The fourth work on "Design Principles for Safety in Human-Robot Interaction" by Manuel Giuliani, Claus Lenz, Thomas Müller, Markus Rickert and Alois Knoll investigates the hardware (and software) requirements in regular industrial robots used in HRI. These robots, used for humanrobot collaborative interaction for more efficient production, are to be equipped with additional sensors to track the human's position and to analyse the human's verbal and nonverbal utterances, while the software that is controlling the robot is especially designed towards safety in the interaction. A robot architecture based on three principles is implied from these studies.

The fifth paper authored by Mathijs Vermeulen and Martijn Wisse on an "Intrinsically safe robot arm" presents a design for a manipulator that is intrinsically mechanically safe. For this the pressure pain thresholds for human skin, a pinching safety constraint is provided that limits the actuator torque, and an impact safety constraint that results in a tradeoff between mass and velocity. To fulfill all constraints, the manipulator introduces a spring balancing system that counteracts gravity in all configurations of the manipulator allowing for extremely low-power DC motors

The sixth article on "The Safety of a Robot Actuated by Pneumatic Muscles-A Case Study" has been authored by Michaël Van Damme, Pieter Beyl, Bram Vanderborght, Rino Versluys, Ronald Van Ham, Innes Vanderniepen, Frank Daerden and Dirk Lefeber. They present the safety study for a lightweight robot actuated by pneumatic muscles which is employed in direct human-robot interaction. Due to its low weight, it allows for excellent hardware safety characteristics. It is also shown that safety can be greatly increased by using Proxy-Based Sliding Mode Control (PSMC). The role of passive compliance in safety is also investigated.

Said Ghani Khan, Guido Herrmann, Tony Pipe, Chris Melhuish and Adam Spiers have written the seventh paper on "Safe Adaptive Compliance Control of a Humanoid Robotic Arm with Anti-Windup Compensation and Posture Control". They consider an adaptive compliance model reference controller using a reference mass-spring-damper system model using an externally sensed force. The scheme is implemented on a 4 degrees of freedom (DOF) humanoid robotic arm. The redundant DOF were used to control the robot motion in a human-like pattern, while actuator saturation issues were addressed by incorporating a novel antiwindup (AW) compensator.

The eighth paper on "Recognizing Hand Grasp and Manipulation through Empirical Copula" by Zhaojie Ju and Honghai Liu recognizes the importance of human hand gesture as a way to deliver messages such as content of safety for avoidance of potential safety hazards. Empirical Copula is introduced for recognizing human motions for the first time using the proposed motion template and matching algorithm. The huge computational cost of Empirical Copula is alleviated by the proposed re-sampling processing. Experiments show that Empirical Copula outperforms other techniques in terms of recognition rate.

The final paper has been written by Emrah Akin Sisbot, Luis F. Marin-Urias, Xavier Broquere, Daniel Sidobre and Rachid Alami on "Synthesizing Robot Motions Adapted to Human Presence-A Planning and Control Framework for Safe and Socially Acceptable Robot Motions". The authors present a robot platform independent motion synthesis framework from planning to execution that is especially designed for a robot that interacts with humans. This framework, composed of PerSperctive Placement, Human Aware Manipulation Planner and Soft Motion Trajectory Planner, generates natural robot motions by taking into account human's safety. The efficacy of the whole system is illustrated in a mobile manipulator robot. 


\section{Conclusions}

This special issue has shown the wide spread interest in an issue of social robotics, safety in human-robot interaction, which clearly requires a suitable engineering and science driven solution. Triggered by the fact that demographic change, technological advancement and improvement in living quality demands the development of service robots which can be used in co-location and collaboration with humans.

Acknowledgements Presenting the wide range of topics and interests on Safety in Human Robot Interaction in the International Journal of Social Robotics has been a great pleasure to the guest editors. We would like to thank the Editor in Chief Shuzhi Sam Ge for inviting us to organize this special issue. In particular, we would like to express our gratitude to Assistant Editor John-John Cabibihan and Gabriela Cisneros, Melika Redolosa and Nathalie Jacobs from Springer for their continuous support and close collaboration.

We also would like to acknowledge all reviewers: Tadej Bajd, Andrew Conn, Manuel Giuliani, Hongmei He, Thomas Holz, Yuancan Huang, Jamaludin Jalani, Said Ghani Khan, Jyrki Latokartano, Claus Lenz, Timo Ilmari Malm, Martin Pearson, Helmuth Radrich, Subramanian Ramamoorthy, Markus Rickert, Emrah Akin Sisbot, Sergey
Skachek, Adam Spiers, Michaël Van Damme, Bram Vanderborght, Juhani Viitaniemi and Martijn Wisse. Their support has been highly valuable for the success of this special issue.

Guido Herrmann (Senior IEEE Member) received the German degree 'Diplom-Ingenieur der Elektrotechnik' (with highest honours) from the Technische Universität zu Berlin, Germany, and the Ph.D.-degree from the University of Leicester, UK, in 1997 and 2001, respectively. From 2001 to 2003, he was a Senior Research Fellow at the Data Storage Institute in Singapore. From 2003 until 2005, he was a Research Associate, Fellow and Lecturer at the University of Leicester. He joined the University of Bristol as a Lecturer in March 2007 and has been since August 2009 a Senior Lecturer. He is leading the Nonlinear Robotics Control Group at the Bristol Robotics Laboratory.

Chris Melhuish is the Director of the Bristol Robotics Laboratory, a partnership between the University of Bristol and the University of the West of England. He is a Fellow of the British Computer Society and is a Chartered Engineer. His research interests include collective mobile robotics, robot-human interaction, neuro-inspired systems and energy autonomy in robots. In addition to his chair at the University of the West of England in Intelligent Autonomous Systems he also holds the chair in Robotics and Autonomous Systems at the University of Bristol. 